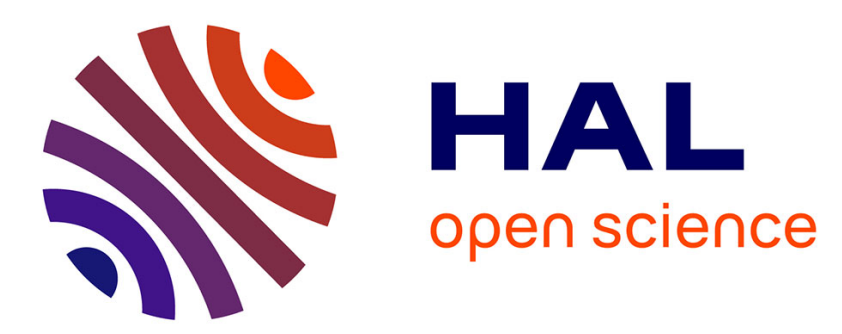

\title{
Influence of stress state and strain rate on the behaviour of a rubber-particle reinforced polypropylene
}

\author{
Virgile Delhaye, Arild Holm Clausen, F. Moussy, Ramzi Othman, Odd Sture
}

Hopperstaad

\section{- To cite this version:}

Virgile Delhaye, Arild Holm Clausen, F. Moussy, Ramzi Othman, Odd Sture Hopperstaad. Influence of stress state and strain rate on the behaviour of a rubber-particle reinforced polypropylene. International Journal of Impact Engineering, 2011, 38 (4), pp.208-218. 10.1016/j.ijimpeng.2010.11.004 . hal-01006979

\section{HAL Id: hal-01006979 \\ https://hal.science/hal-01006979}

Submitted on 11 Mar 2018

HAL is a multi-disciplinary open access archive for the deposit and dissemination of scientific research documents, whether they are published or not. The documents may come from teaching and research institutions in France or abroad, or from public or private research centers.
L'archive ouverte pluridisciplinaire HAL, est destinée au dépôt et à la diffusion de documents scientifiques de niveau recherche, publiés ou non, émanant des établissements d'enseignement et de recherche français ou étrangers, des laboratoires publics ou privés. 


\title{
Influence of stress state and strain rate on the behaviour of a rubber-particle reinforced polypropylene
}

\author{
V. Delhaye ${ }^{\mathrm{a}, \mathrm{b}, \mathrm{c}, \mathrm{d}, *}$, A.H. Clausen ${ }^{\mathrm{a}, \mathrm{b}}, \mathrm{F}$. Moussy $^{\mathrm{c}}$, R. Othman ${ }^{\mathrm{d}}$, O.S. Hopperstad ${ }^{\mathrm{a}, \mathrm{b}}$ \\ a Structural Impact Laboratory (SIMLab), Centre for Research-Based Innovation, Norwegian University of Science and Technology (NTNU), NO-7491 Trondheim, Norway \\ ${ }^{\mathrm{b}}$ Department of Structural Engineering, Norwegian University of Science and Technology (NTNU), NO-7491 Trondheim, Norway \\ ${ }^{\mathrm{c}}$ Renault Materials Engineering Dept., Technocentre Renault-TCR LAB 050, France \\ ${ }^{\mathrm{d}}$ Institut de Recherche en Génie Civil et Mécanique (GeM), Ecole Centrale de Nantes, F-44321 Nantes cedex 3, France
}

\begin{abstract}
This article presents an experimental investigation of a ductile rubber-modified polypropylene. The behaviour of the material is investigated by performing tension, shear and compression tests at quasistatic and dynamic strain rates. Subsequently, scanning electron microscopy is used to analyse the fracture surfaces of the tension test samples, and to relate the observed mechanical response to the evolution of the microstructure. The experimental study shows that the material is highly pressure and strain-rate sensitive. It also exhibits significant volume change, which is mainly ascribed to a cavitation process which appears during tensile deformation. Assuming matrix-particle debonding immediately after yielding, the rubber particles might play the role of initial cavities. It is further found that the flow stress level is highly dependent on the strain rate, and that the rate sensitivity seems to be slightly more pronounced in shear than in tension and compression. From the study of the fracture surfaces it appears that the fracture process is less ductile at high strain rates than under quasi-static conditions.
\end{abstract}

Keywords: Thermoplastics, Mechanical tests, Dilation, Voids, Scanning electron microscopy

\section{Introduction}

Thermoplastics have during the last couple of decades found their use in an increasing number of applications, also involving the automotive industry. Typical examples from this field are bumpers and dashboards. Common for these components is that they have to be designed for possible crash situations. The reason why thermoplastics are so interesting for such applications is their excellent compromise between low density and mechanical properties relevant for energy absorption. On the other hand, today's main tool when designing such parts is the finite element method. Accurate numerical predictions of the response caused by e.g. an impact event require a material model that is able to represent the most important features of the thermoplastic at hand. This is a challenge in the available commercial finite element codes. Obviously, development of suitable models for thermoplastics demands good knowledge of the mechanical behaviour and preferably also the mechanisms at the meso-scale.

\footnotetext{
* Corresponding author. Structural Impact Laboratory (SIMLab), Centre for Research-Based Innovation, Norwegian University of Science and Technology (NTNU), NO-7491 Trondheim, Norway. Tel.: +47 73591300.

E-mail address: virgile.delhaye@ntnu.no (V. Delhaye).
}

This paper presents results from mechanical tests on a polypropylene block copolymer, which is applied by automotive companies in bumpers and other parts. Within this field of engineering, it is common to modify the materials with rubber phases during the injection process to increase the ductility of the material when exposed to high strain rates. These rubber phases have therefore an influence on the macroscopic behaviour of the material. In particular, knowledge of the behaviour of these materials under different stress states and strain rates is required because they are applied in complex car components which may be subjected to various loading situations. Material tests reveal such information, and serve also to provide insight in relevant features to cover when a material model for thermoplastics is to be developed.

Mechanical testing of thermoplastic materials demands in general a more complex setup than what is necessary for tests on metals. One reason is the intrinsic softening effects appearing at the early stages of yielding, inducing instabilities in the specimens and subsequently a non-homogeneous deformation field [1,2]. Further, thermoplastics may exhibit volume changes when deformed [3], which can often be related to craze growth and subsequent cavitations in the amorphous phases. Besides, a strong rate dependence is often reported [4,5] which may involve thermo-mechanical effects in some cases [6,7]. In the case of rubber blended polymers, 
the presence of rubber phases has also an effect on the general behaviour of the material. Several authors have dealt with the behaviour of cavitated polymers [8,9,10], and claim that the cavitation occurring in such materials is an important dissipation process during deformation. From a microstructural point of view, cavitation is generally induced by debonding of rubber particles. The usual tool applied for investigation of this topic is scanning electron microscopy (SEM) of the fracture surfaces, visualizing the role of the particles [11,12]. However, it is more difficult to find information on the influence of the strain rate on the cavitation behaviour, and on the interrelation between microstructural evolution of the material and the observed mechanical response under different states of stress as it exists when a polymeric car component is subjected to crash.

Experimentally, the features described above call for full-field strain measuring techniques. We have used digital image correlation (DIC) in the material tests presented in this paper. A random speckled pattern is applied at the gauge area of the specimens before the test. The evolution of the speckled pattern when the material is deformed is acquired by a digital camera. Subsequently, the DIC software uses the camera pictures to determine the displacement field and thereafter to calculate the deformation gradient field and the in-plane strain field with reference to the original configuration [13].

In the current paper, mechanical tests in tension, shear and compression are performed to investigate the effect of stress state on the response of the material. The material was delivered as injection-moulded plates, and the possible anisotropy of the mechanical properties is therefore also evaluated. The tests are carried out at different strain rates between $10-3$ and, where the higher level corresponds approximately to the strain rate experienced by an automotive part in a crash situation. SEM analyses of the fracture surface were also carried out, as this enables an evaluation of the microstructural mechanisms, which is a key to understand the mechanical properties of the material at a macroscopic level. The main contribution of the paper is to describe the mechanical behaviour of a rubber-modified polypropylene copolymer under different stress states and strain rates relevant for structural impact applications. Full-field measuring techniques were employed in the strain calculations, and the observed macroscopic behaviour was partly explained by microscopic investigations.
The paper is organised as follows. Section 2 provides an overview of the experimental programme, involving procedures for tension, compression and shear tests at different rates as well as SEM analysis. The results from the mechanical tests and SEM study are presented in Sections 3 and 4, respectively, while Section 5 provides a discussion of the findings. The concluding remarks in Section 6 close the paper.

\section{Material and methods}

\subsection{Material description}

The material investigated in this paper is a (PP-EPR) impact block copolymer, consisting of a polypropylene (PP) matrix and ethylene-propylene rubber (EPR) particles. The fractions are respectively 78 and $22 \mathrm{wt} \%$. The degree of crystallinity of the PP phase has been estimated to $50 \%$. The EPR particles consist of $50 \%$ propylene and $50 \%$ ethylene. Because the matrix and particles are not miscible, the EPR phase can be considered as rubber inclusions into a PP matrix. Mineral inclusions are also present in low quantity (0.5\%). The material has good mechanical properties for crash applications and exhibits a very high ductility.

The material was injection-moulded into plates of $3 \mathrm{~mm}$ thickness. Both the temperature of the mould and the flow were kept constant during the process to avoid residual stresses. The subsequent cooling was slow enough to ensure that the temperature could be considered homogeneous in the plate during the whole process. Thus, the plates were supposed to have homogeneous material properties. This issue was checked in the tension loading mode, see Section 3.1.

\subsection{Experimental setup for mechanical tests}

Mechanical tests were carried out in tension, compression and shear at several strain rates at room temperature to establish the mechanical behaviour relevant for crash situations. Most of the tests were monitored with a digital camera, providing pictures for a subsequent determination of the strain field applying digital image correlation (DIC).

In tension, modified ISO527b specimens were used for all strain rates, see Fig. 1a. A small imperfection was machined at both sides of the specimen and near the centre of its gauge section to localise



C

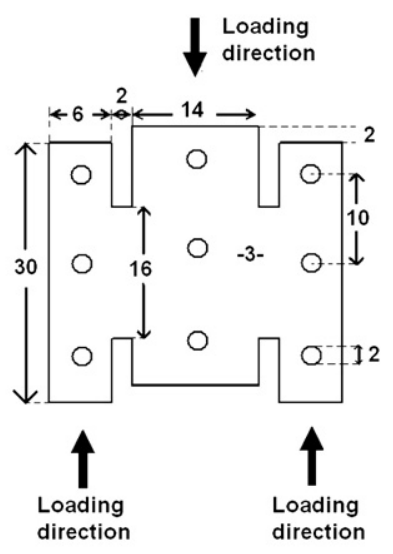

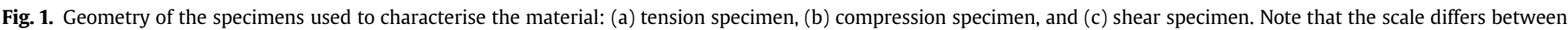
the three parts of the figure. 




Fig. 2. Nominal stress-strain curves in tension for different sample orientations. Nominal strain rate: $10^{-2} \mathrm{~s}^{-1}$.

the deformation and thus get a better resolution of the strain measurements. The imperfection had a shape of a curved fillet, with a depth of $0.25 \mathrm{~mm}$ and a length of $15 \mathrm{~mm}$. A numerical study was carried out to optimise the geometrical shape and dimensions of the imperfection to ensure that it does not introduce any significant stress triaxiality. Similar modifications of tension test specimens are reported in the literature $[3,14]$. All tension tests presented herein were performed with a servo-hydraulic machine, covering average strain rates from $10-3$ to. For both force and picture acquisition the sampling frequency was $1 \mathrm{~Hz}$ at, $10 \mathrm{~Hz}$ at while it was $4000 \mathrm{~Hz}$ at $100 \mathrm{~s}^{-1}$.

The geometry of the compression specimens is given in Fig. 1b. The design was defined by a sufficient low slenderness to avoid buckling. The dimensions of the specimen were rather small because of the limitation defined by the thickness of $3 \mathrm{~mm}$ of the injection-moulded plates. For the quasi-static strain rates $\left(10^{-2} \mathrm{~s}^{-1}\right)$, a hydraulic testing machine was used, while a directimpact Hopkinson bar [15] was employed at the higher strain-rate level $\left(100 \mathrm{~s}^{-1}\right)$. In this setup, only the transmission bar was used. The striker impacted the specimen directly. Hence, most of the striker kinetic energy was used to deform the specimen. Three strain gauges were cemented on the bar. The dynamic stress and strain data were calculated using the wave separation technique developed by Bussac et al. [16]. In the quasi-static compression tests at $10^{-2} \mathrm{~s}^{-1}$, the sampling rate of force and camera acquisition was $10 \mathrm{~Hz}$. For the elevated strain rate of $100 \mathrm{~s}^{-1}$ the sampling rate of the force acquisition was $10,000 \mathrm{~Hz}$, while it was $30,000 \mathrm{~Hz}$ for the digital camera.

Fig. 1c shows the geometry of the shear sample. It is a double shear specimen where small notches have been machined at each side of the gauge area to localise the shear deformation. The shape of the specimen is rather similar to that proposed by G'Sell et al.



Fig. 3. Nominal stress-strain curves in tension for different specimens taken at the same location in different plates. Nominal strain rate: $10^{-2} \mathrm{~s}^{-1}$.

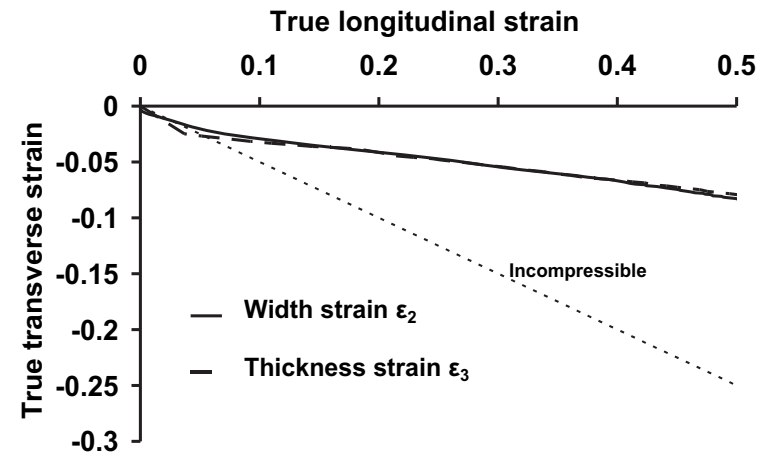

Fig. 4. Evolution of the in-plane and through-thickness transverse strains as function of longitudinal strain during tensile testing. Nominal strain rate: $10^{-2} \mathrm{~s}^{-1}$.

[17]. Their buckling and load capacity criteria are satisfied, but the thickness in the gauge section was not reduced. This simple geometry provides predominant shear state of stress for moderate strains, i.e. less than 0.3. After that, the normal stresses initiated along the grips become too significant to consider the specimen being in a shear state. A hydraulic machine was used in the quasistatic tests $\left(5 \cdot 10^{-3} \mathrm{~s}^{-1}\right)$. For moderate strain rates $\left(200 \mathrm{~s}^{-1}\right)$, a purpose-made setup was made to adapt the shear specimens into the compression direct-impact Hopkinson bar. The intrinsic constitution of the material and the localisation process acting when the material is stretched cause a variation in the instantaneous strain rate from to during the dynamic shear test. Average values of the shear strain rate are therefore given. At, the sampling rate was $100 \mathrm{~Hz}$ for the force acquisition and $20 \mathrm{~Hz}$ for the camera, while the rates were increased to respectively $10,000 \mathrm{~Hz}$ and $25,000 \mathrm{~Hz}$ in the Hopkinson bar tests $\left(200 \mathrm{~s}^{-1}\right)$.

\subsection{Strain measurements}

During the tests, the in-plane strain field was acquired using digital camera images and digital image correlation (DIC). The DIC software determines the in-plane Green-Lagrange strains $E_{i j}$ from the sequence of digital images, where $i, j=1,2$ or $i, j=1,3$ depending on which surface of the sample being investigated. The indices $i, j=1,2,3$ are defined from the base vectors $\mathrm{e}_{1}$ shown in Fig. 1 .

For uniaxial tension and compression, it is convenient to relate the principal values of $E_{i j}$ to the principal values of the Hencky (or logarithmic) strain tensor $\varepsilon_{i j}$. To this end, a material volume element with dimensions $l_{0 i}$ and $l_{i}$ along the $x_{i}$-axis in the initial and current configurations, respectively, is considered. It is further assumed that the base vectors $e_{1}$ are along the principal directions of the

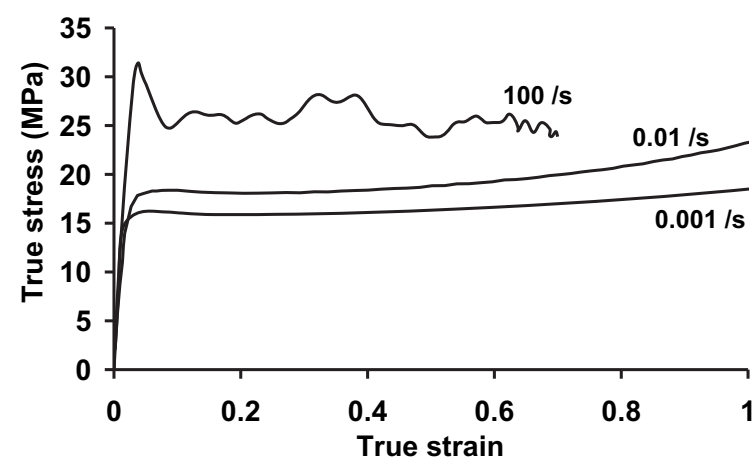

Fig. 5. True stress-strain curves in tension for different strain rates. 




Fig. 6. Evolution of the transverse strains in tension for different strain rates.

Green-Lagrange strain tensor $E_{i j}$. Then the relation between the principal values $\varepsilon_{i}$ and $E_{i}(i=1,2,3)$ of respectively the Hencky and Green-Lagrange strain tensors reads

$\varepsilon_{i}=\ln \left(\frac{l_{i}}{l_{0 i}}\right)=\ln \left(\sqrt{1+2 E_{i}}\right)=\frac{1}{2} \ln \left(1+2 E_{i}\right), i=1,2,3$

It is further necessary to quantify the volume changes during the deformation process. The volumetric Hencky strain is defined by

$\varepsilon_{V}=\ln \left(\frac{V}{V_{0}}\right)=\ln \left(\frac{l_{1} l_{2} l_{3}}{l_{01} l_{02} l_{03}}\right)=\varepsilon_{1}+\varepsilon_{2}+\varepsilon_{3}$

where $V_{0}$ is the initial volume of the material and $V$ is the volume in the deformed configuration. In some cases, the nominal strains $e_{i}$ will be used in presenting the results from the uniaxial tension and compression tests, and these strains are defined by

$e_{i}=\frac{l_{i}-l_{0 i}}{l_{0 i}}=\exp \left(\varepsilon_{i}\right)-1, i=1,2,3$

It is noted that $\varepsilon_{i}$ are often referred to as true strains to distinguish them from nominal strains, which refer to the initial length of the considered material element.

For the case of double shear, the shear strain $\gamma$ can be expressed in terms of the in-plane components of the Green-Lagrange strain tensor by

$\sin (\gamma)=\frac{2 E_{12}}{\sqrt{1+2 E_{11}} \sqrt{1+2 E_{22}}}$

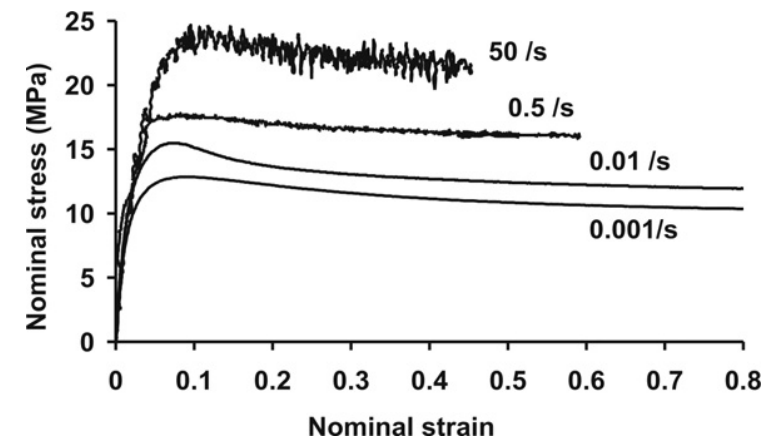

Fig. 7. Nominal stress-strain curves in tension for different strain rates. The end of the curves does not correspond to fracture.



Fig. 8. True stress-strain curves in compression for different strain rates. Absolute values of stress and strain are plotted in the figure.

\subsection{Stress calculation}

The nominal stress $s_{1}$ in the $x_{1}$-direction in tension and compression is defined as

$s_{1}=\frac{P}{S_{0}}$

where $P$ is the force applied to the specimen and $S_{0}$ is the crosssection area in the initial configuration. Using information from the full-field strain measurements, the calculation of the Cauchy (or true) stress $\sigma_{1}$ in uniaxial tension and compression is straightforward, i.e.

$\sigma_{1}=\frac{P}{S}=\frac{P}{l_{2} l_{3}}=\frac{P}{S_{0}} \exp \left(-\varepsilon_{2}-\varepsilon_{3}\right)$

where $S$ is the cross-section area in the current configuration. The subscripts 1, 2 and 3 still refer to the base vectors shown in Fig. 1, and it is assumed that the principal directions of stress and strain coincide. Under uniaxial loading, i.e. in compression and tension, the assumption of transverse isotropic material leads to $\varepsilon_{2}=\varepsilon_{3}$, and it follows that

$\sigma_{1}=s_{1} \exp \left(-2 \varepsilon_{2}\right)$

The assumption of transverse isotropy is evaluated in Section 3.1.

In the case of double shear specimens, the mean shear stress is obtained from

$\tau=\frac{P}{2 S_{V}}$

where $P$ is the force acting on the specimen and $S_{V}$ is the gauge area where the shear stress is applied. The factor 2 in the denominator is



Fig. 9. Evolution of the transverse strain as function of longitudinal strain during compression testing at two strain rates. Note that Absolute value of the longitudinal strain is used. 




Fig. 10. Shear stress-strain curves at two different shear strain rates.

related to the double shear specimen because the load $P$ acts on two symmetric areas of size $S_{V}$, see Fig. 1c.

\subsection{Scanning electron microscopy}

Fracture surfaces of several tensile specimens from the quasistatic and dynamic test programme were analyzed with a scanning electron microscope (SEM) to reveal the damage and fracture mechanisms of the material. A JSM-6700F instrument from JEOL was used in the investigation. For reference, micrographs were also taken from samples of the virgin material. These samples were broken after being cooled in liquid nitrogen.

Preparation of the samples for SEM had to be done with care to avoid any damage of the material. Also, thermoplastics are complex to analyze in a SEM because of their very low electrical conductivity which can trigger artefacts and even damage the material. To avoid these difficulties, the samples were first cleaned of intrusive particles using an ultrasonic technique. Thereafter, the samples were metallised in a gold plasma gas to increase their conductivity and reduce the accumulated charges at the surface of the specimen when submitted to the electronic beam.

\section{Results}

\subsection{Evaluation of isotropy and experimental scatter}

The material was injection-moulded into $3 \mathrm{~mm}$ thick plates as described in Section 2.1. Although precautions were taken during the moulding process to avoid material anisotropy, there may be a coupling between process and material properties. Tensile samples were therefore machined at three different in-plane angles $\left(0^{\circ}, 45^{\circ}, 90^{\circ}\right)$ with respect to the flow direction during the injection moulding in order to check if there is any in-plane anisotropy.

Fig. 2 shows the obtained nominal stress-strain curves. The longitudinal orientation (L) corresponds to the flow direction, whereas the transverse direction (T) is perpendicular to the flow. The third orientation (45) is diagonal, i.e. has an angle of $45^{\circ}$ with respect to the flow direction. The response appears to be similar in the three directions, indicating planar isotropy.

The scatter between different plates was investigated by machining samples from the same location in three different plates. The specimens were tested in tension at $10^{-2} \mathrm{~s}^{-1}$, and the nominal stress-strain curves are provided in Fig. 3. Clearly, the batch repeatability seems to be good.

The transverse deformation of the material in tension was also checked to evaluate any possible flow anisotropy. The samples were taken at the same location in the middle of the plate. Two groups of specimens were investigated. For the first group of specimens, the front of the samples defined by the $\left(e_{1}, e_{2}\right)$-plane was monitored with the digital camera, whereas the side of the samples defined by the $\left(e_{1}, e_{3}\right)$-plane was studied for the second group. The coordinate system is as defined in Fig. 1. Again, the experimental repeatability was good. Fig. 4 shows the evolution of the transverse strains versus the longitudinal strain for a representative test. The special case of an incompressible material is also plotted for comparison; for such a material, the evolution of $\varepsilon_{2}$ and $\varepsilon_{3}$ as function of $\varepsilon_{1}$ is linear with a slope of -0.5 . The measured evolution of the transverse strains is similar for the two groups of specimens indicating that the plastic flow of the material is rather isotropic. Consequently, all true normal stresses reported in this paper are calculated with Eq. (7), utilising the transverse in-plane strain $\varepsilon_{2}$.

The evolution of the transverse strains shows clearly that the volume increases during stretching when the longitudinal strains exceed 0.05. Moreover, the fact that the transverse strains are much smaller than they would be in the incompressible case implies that these dilations are significant. The volume change occurring when the strain exceeds 0.05 corresponds experimentally to the initiation of plastic deformations and whitening of the material. At strains below 0.05 , the slopes of the experimental curves are rather close to that in the incompressible case. Poisson's ratio was estimated to be between 0.4 and 0.5 depending on which transverse strain component was used in the calculation. This variation is attributed to uncertainties in the strain measuring technique because of the
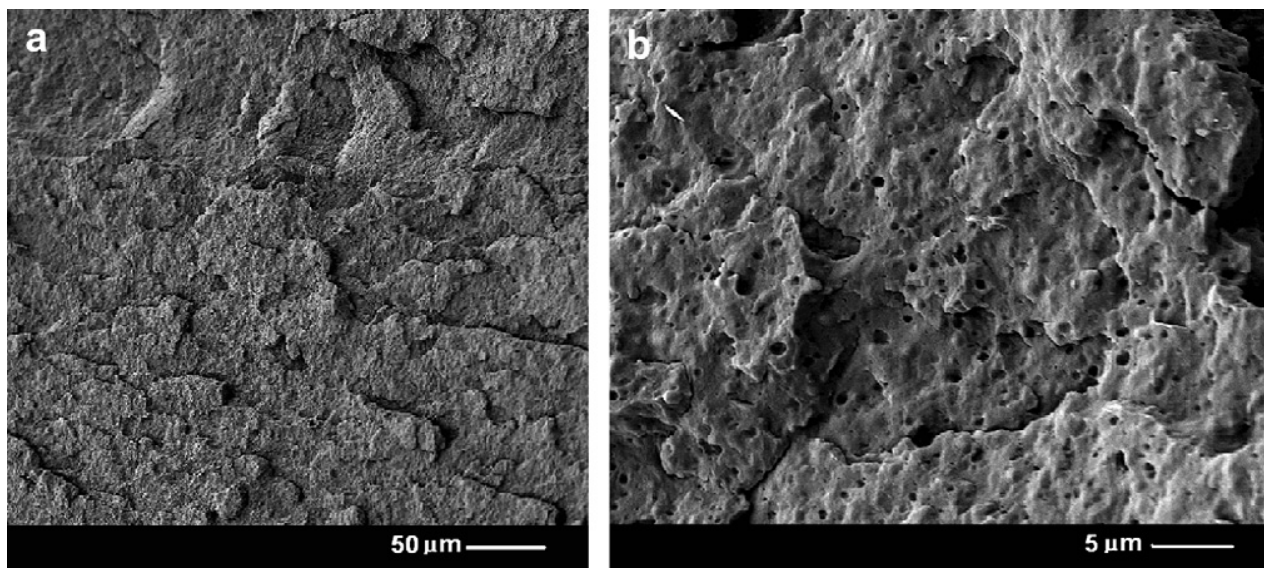

Fig. 11. Virgin material fractured at $-196{ }^{\circ} \mathrm{C}$ in tension: (a) overview, and (b) magnification of a part of micrograph (a). 


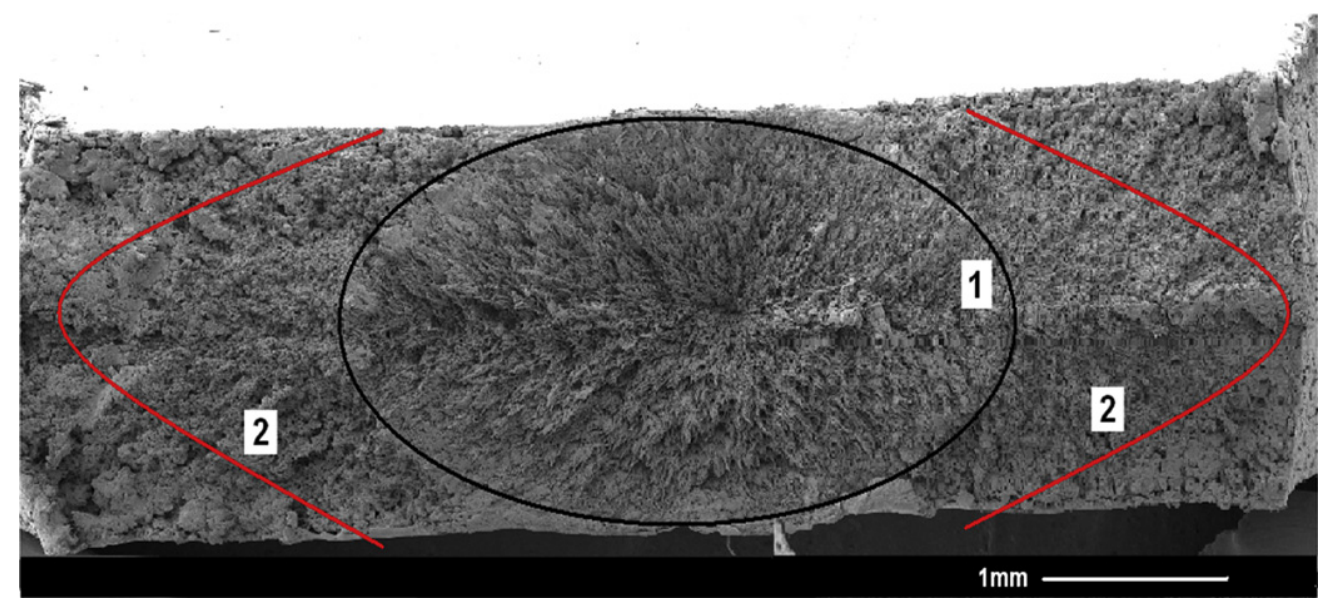

Fig. 12. Fracture surface of a tensile specimen deformed at quasi-static strain rate, identifying the initiation area (1) and the propagation area (2).

low thickness of the specimens and very small deformations in the elastic range. The accuracy improves when the strains increase.

\subsection{Tension tests}

Fig. 5 shows the true stress-strain curves of the material at three different strain rates in tension. The material exhibits strain hardening due to molecular stretching at quasi-static strain rates. For the dynamic case, however, yielding starts by a peak immediately followed by a severe drop. The ensuing strain hardening for large deformations is less than in the quasi-static case. The oscillations occurring in the stress-strain curve for the dynamic case is most probably due to the inertia of the force sensor and the grips' disposal [18]. The use of a disposal is not avoidable in tension at such strain rates. Oscillations induce uncertainties on the force measurements, but the oscillations have a very particular signature, and their amplitude remains acceptable. Here we choose to represent the raw data, but it is possible to smooth the data by a moving average technique or some filtering. The full-field strain measurements were not impacted by the oscillations on the force signal.

Fig. 6 shows the evolutions of the transverse strains for the different strain rates, including also the case of an incompressible material. It appears that the transverse strains and hence the volume change in tension do not seem to depend on the strain rate. Nominal stress-strain curves for four strain rates are presented in Fig. 7. These results were obtained without the use of full-field deformation measurements. The evolution of the yield stress with the strain rate is similar in both test series reported in Figs. 5 and 7, indicating that a strain-rate characterization of a polymer material does not necessarily require an experimental setup involving DIC. Nominal yield stress data may suffice.

\subsection{Compression tests}

The results of the compression tests are given as true stress-strain curves in Fig. 8 for two strain rates. The behaviour differs from what was observed in tension. In particular, the flow stress appears to be significantly higher. In the quasi-static case, i.e. at strain rate $10^{-2} \mathrm{~s}^{-1}$, the flow stress changes from $18 \mathrm{MPa}$ in tension to $28 \mathrm{MPa}$ in compression, which represents a ratio of 1.5 . Another interesting observation from Fig. 8 is that the shape of the stress-strain curve after the yield point clearly differs between the static and dynamic cases. The strain hardening observed in the static case does not seem to be present under dynamic loading.

Still considering the compression tests, the evolution of the transverse strain is plotted as function of the longitudinal strain in Fig. 9. The response of an incompressible material is also here plotted for comparison. Like in tension, the transverse strain has a similar evolution for both strain rates, indicating that the volume change in compression does not depend on strain rate. Another observation from Fig. 9 is that the experimental curves are located under the line representing the behaviour of an incompressible material, which means that the volume decreases. These changes
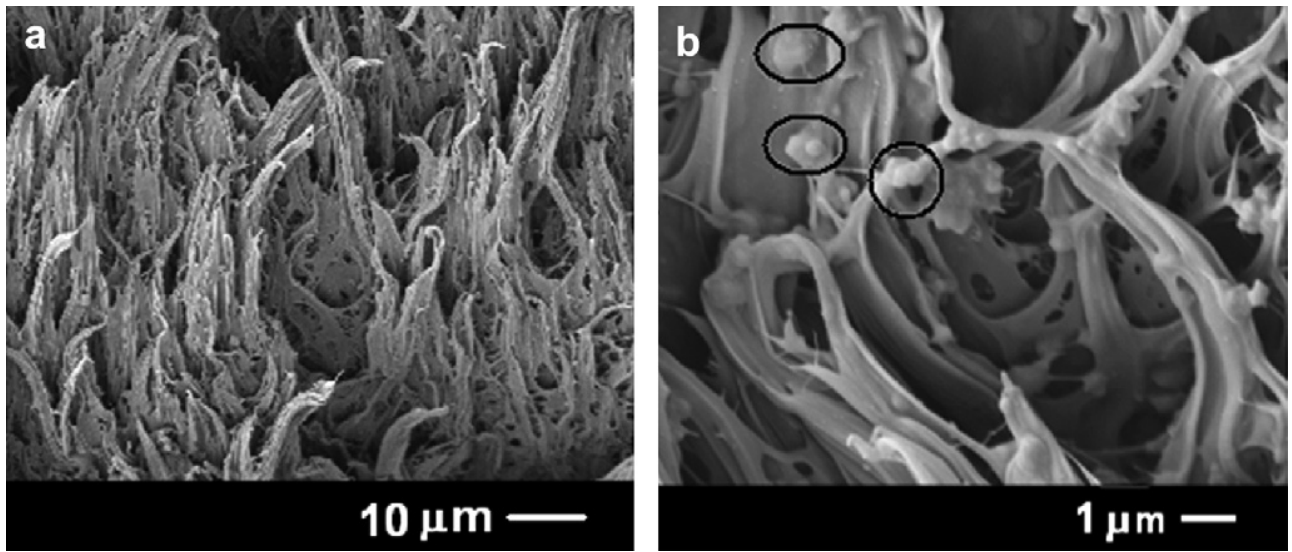

Fig. 13. High magnification micrographs of the initiation area (1) of Fig. 12, showing a fibril-like structure: (a) overview, and (b) magnification of a part of micrograph (a). 

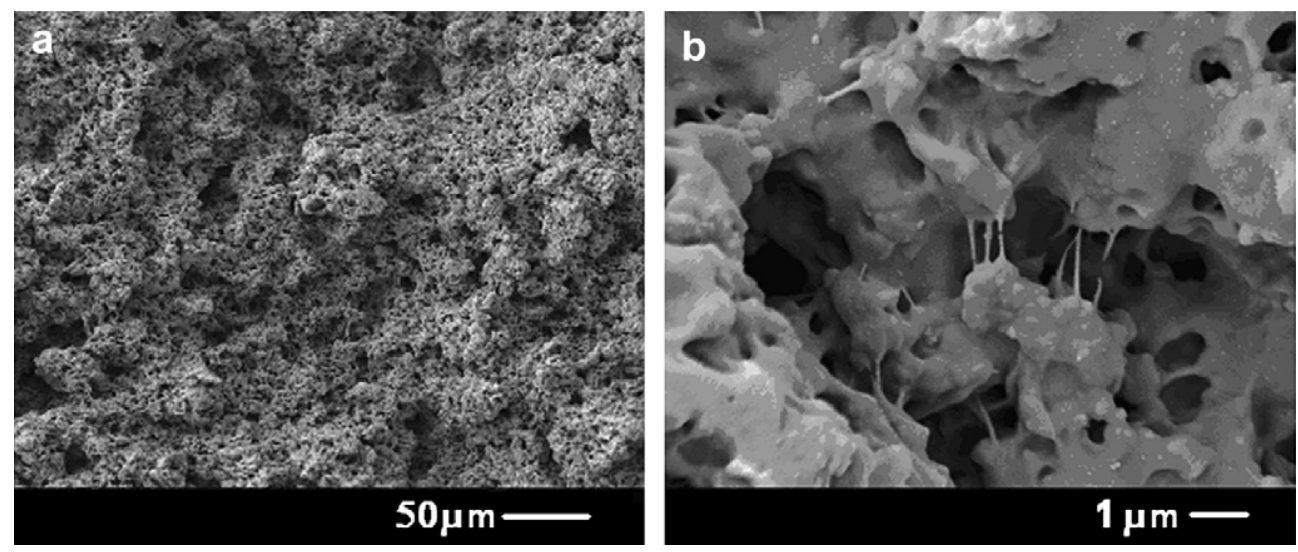

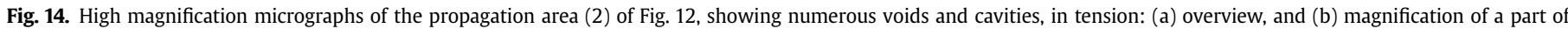
micrograph (a).

are, however, smaller than in the tensile case because the curves are closer to the line representing the incompressible response. A similar behaviour has been observed in several cavitated polymer blends $[19,20]$ and is probably related to the kinetics of void evolution in the material.

\subsection{Shear tests}

The behaviour in shear is given in Fig. 10, showing shear stress-strain curves at a quasi-static and dynamic strain rates. The shape of the curves is rather similar to those observed in tension and compression, see Figs. 5 and 8, although the transition from the initial elastic part to the plastic flow regime appears to be more gradual in the case of shear. Also, the strain rate sensitivity seems even more pronounced in shear than it is in the two other loading modes. The level of the flow stress plateau is doubled by increasing the shear strain rate from $0.005 \mathrm{~s}^{-1}-200 \mathrm{~s}^{-1}$, while a similar increase of four decades of strain rate in tension or compression yields an increase in flow stress with a factor around 1.6. It should be noted, however, that we experienced some difficulties in obtaining a constant shear strain rate in the dynamic tests. The reported dynamic strain rate of $200 \mathrm{~s}^{-1}$ in Fig. 10 was an average rate, while the instantaneous local strain rate at the onset of yielding had a maximum value of $500 \mathrm{~s}^{-1}$.

The test results from Figs. 5 to 10 show that the mechanical response of the material is very dependent on the state of stress and the strain rate. It is further established that there is a significant change of volume when the material deforms in tension and compression. Thus, information about the microstructure of the material and its evolution when deformed is relevant for an improved understanding of the underlying deformation mechanisms.

\section{Results from SEM analysis}

\subsection{SEM micrographs of undeformed material}

The fracture surfaces were investigated with a scanning electron microscope as described in Section 2.5. As a reference for the brittle fracture morphology and to evaluate the microstructure of the virgin material, micrographs were also obtained from a nondeformed sample that was broken after being cooled in liquid nitrogen at $-196{ }^{\circ} \mathrm{C}$, see Fig. 11. It is evident from Fig. 11b that cavities are present. This may explain some of the mechanical properties, and in particular, the pressure dependency and volume change. A reasonable assumption could be that the small cavities might correspond to the location of the rubber phases in the PP matrix, which debonded or cavitated during the fracture process in liquid nitrogen or during the subsequent sample preparation. This debonding is explained by a decrease of the adhesion properties of the rubber phases with the matrix. An analysis of the fracture surfaces indicates an initial void density of approximately $20 \%$.


Fig. 15. (a) Low magnification micrograph of the fracture surfaces in tension at dynamic strain rate. (b) Magnification of the area with ductile fracture. 

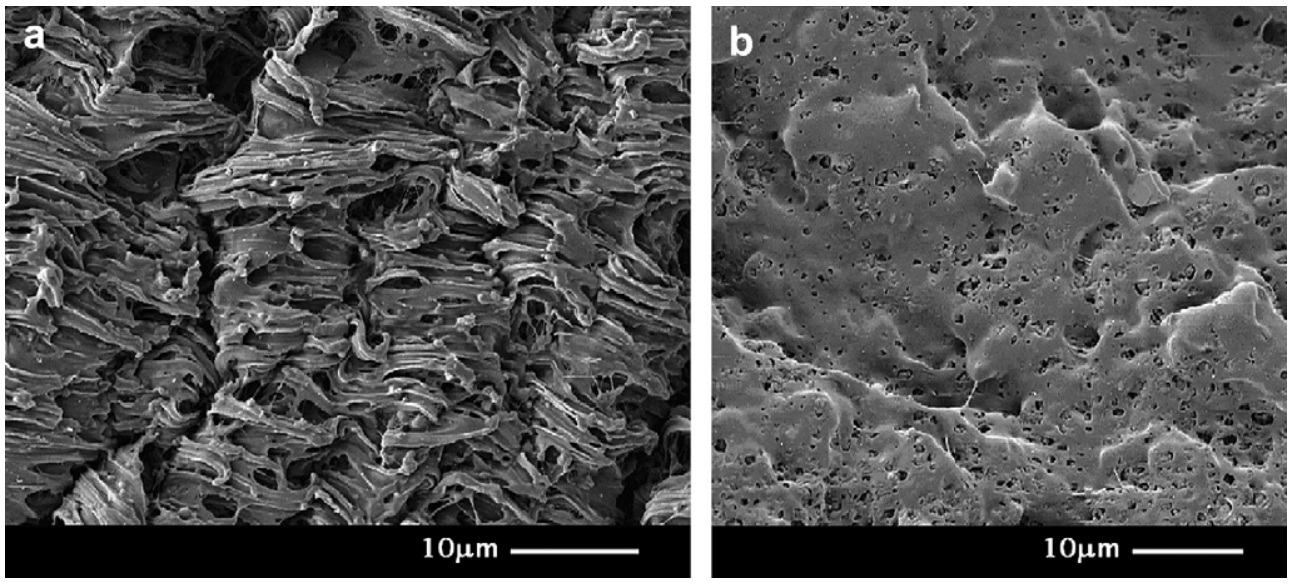

Fig. 16. High magnification micrographs of the fracture surfaces in tension at dynamic strain rate: (a) initiation area, and (b) propagation area.

\subsection{SEM micrographs of tension samples}

Fig. 12 shows the through-thickness fracture surface of a specimen subjected to quasi-static tension. Two different fracture morphologies can be identified, corresponding to the initiation area (1) and propagation area (2). The initiation area (1) was not always located in the middle of the sample, but always remained ductile. Long stretched ligaments can be seen, which are characteristic of ductile cavitated polymers. The surrounding area (2) corresponds to a fast brittle propagation of the crack with cleavage morphology. Such morphologies have previously been observed in blended polymers in the same conditions $[8,9]$.

A close-up look of the ductile area (1) in Fig. 12 is given in Fig. 13. The fibril-like structure is clearly seen. This filament structure is characteristic of the interaction existing between cavities and the PP matrix. An explanation could be formation of micro-necks during tensile deformation, which according to Galeski [21] and Peterlin [22] is favoured by the presence of voids. In this case, voids are initiated by rubber phases debonding from the matrix. Another interesting observation in the area with ductile fracture is the presence of spheroids; see the encircled areas in Fig. 13b. They have the same size as the small voids observed in the virgin material, see Fig. 11b. The material shown in Figs. 12 and 13 has not been subjected to any cooling. The fracture is therefore ductile and the particles are likely to still be present because of a higher degree of cohesion with the PP matrix, recognizable as spheroids in Fig. 13b. Another possibility is that the visible particles in Fig. 13b could be the mineral inclusions initially present in low quantity in the material.

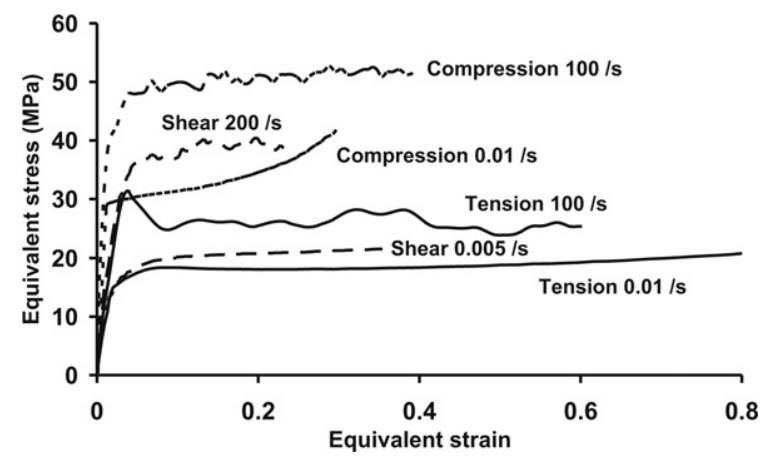

Fig. 17. Equivalent stress-strain curves obtained at different deformation modes and strain rates.
Fig. 14 presents a magnification of the propagation area (2) identified in Fig. 12. A lot of cavities and voids are observed. Their size has increased significantly from the non-deformed to the deformed specimens. This is related to the macroscopic volume increase measured during tensile testing. The ligament structure that was observed in the initiation area, see Fig. 13, is not present in Fig. 14.

The effect of the strain rate on the microstructure of the deformed material has also been investigated. The fracture morphology of a specimen fractured at a strain rate around $50 \mathrm{~s}^{-1}$ is shown in Fig. 15, and the fracture surfaces differ clearly from those obtained at quasi-static test conditions, see Fig. 12. Also here, different types of morphology are found. A ductile area with formation of fibrils is present in the right-hand part of Fig. 15a. The ductile area is now much smaller than it was in the quasi-static test. In this particular case, fracture was initiated at the border of the sample. This area is magnified in Fig. 15b. Moreover, a brittle area with voids and cleavage morphology can be observed in the lefthand part of Fig. 15a, and there is also an area with intermediate morphology.

Fig. 16 shows high magnification micrographs of the same tension specimen, addressing respectively the initiation and propagation area. In a similar way as under quasi-static loading conditions, the fracture initiation is ductile with presence of long pulling filaments, see Fig. 16a. These filaments are shorter in the dynamic case than they were at quasi-static test conditions. The

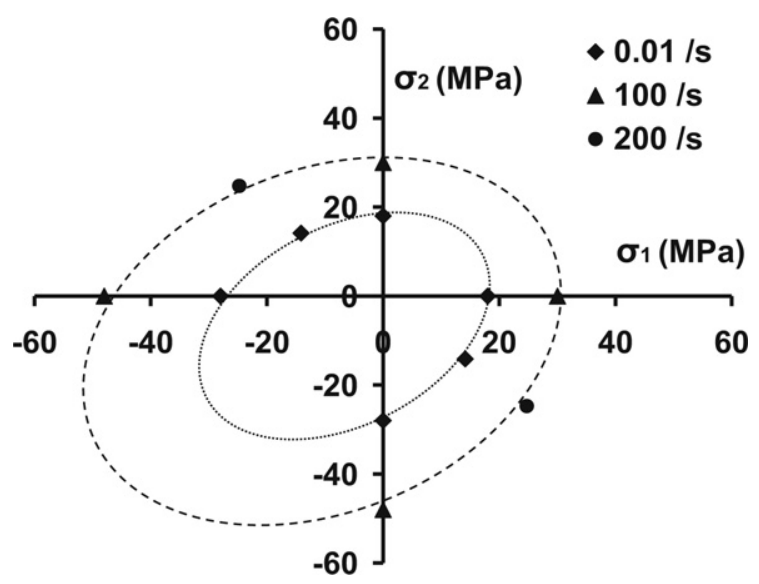

Fig. 18. Representation of the yield loci in the $\left(\sigma_{1}, \sigma_{2}\right)$-plane for different orders of strain rate. 


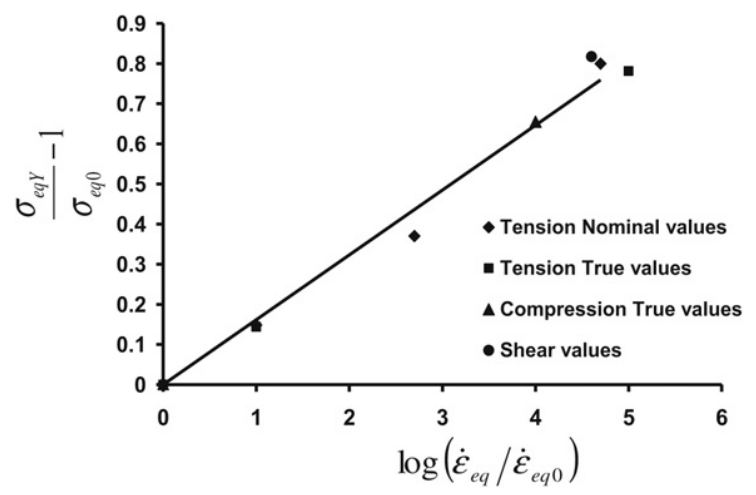

Fig. 19. Evolution of the yield stress as function of strain rate.

most significant differences, however, can be observed by magnifying the propagation area, see Fig. 16b. The cavities are smaller than in the quasi-static case, and this is possibly a strain-rate effect. At high strain rates, the cavities do not have time to grow, as they would do under slow test conditions. The result is that the material breaks at a lower deformation. The morphology of the cavities and of the material around them is also very different from that of the quasi-static case. The shapes of the cavities are less regular and the edges seem less sharp. An explanation for this could be the significant self-heating of the material at this strain rate during deformation process, which changes the morphology as the fracture occurs. The fracture surface appears indeed very similar to those observed by van der Waal and Gaymans [23] for polymerrubber blends under dynamic conditions.

\section{Discussion}

Fig. 17 shows the mechanical response of the material at different states of stress and strain rates. For the purpose of comparison, the values are plotted using equivalent stress and strain measures. In uniaxial tension and compression, $\sigma_{e q}=\left|\sigma_{1}\right|$ and $\varepsilon_{e q}=\left|\varepsilon_{1}\right|$, while in shear, $\sigma_{e q}=\sqrt{3 \tau}$ and $\varepsilon_{e q}=\gamma / \sqrt{3}$. Significant differences of behaviour between the three states of stress represented can be noticed. It is observed that the strain hardening in tension is rather weak. From a microstructure point of view, this is linked to the presence of the particles in the virgin material which are likely to debond at the early stage of the deformation process and subsequently play the role of pre-existing cavities. They represent about $20 \%$ of the total volume. The growth of cavities induces softening and damage which counteract the intrinsic strain hardening of the material. Similar behaviour has already been observed for filled thermoplastics in tension $[4,5,12,13]$, and in particular, the influence of the volume fraction of particles which tends to increase the softening effect.

The material is pressure dependent as the flow stress increases significantly from tension via shear to compression. This is evidence indicating that the material is subjected to a cavitation process in tension. Several authors have discussed the role of the pre-cavitation on the different response of polymers under different states of stress, e.g. Pawlak and Galeski [24]. Another explanation could also be a significant frictional effect in this material due to the sliding between molecular chains when stretched [25].

The material is highly rate sensitive in all deformation modes. To illustrate this, yield loci in the plane of principal stresses $\left(\sigma_{1}, \sigma_{2}\right)$ are indicated for rates of order $10^{-2}$ and $100 \mathrm{~s}^{-1}$ in Fig. 18. All yield stresses addressed as points in the figure correspond to the local maxima point of their respective stress-strain curves. In the case where no local maximum is observed on the true stress-strain curves in tension, the yield stress is defined using nominal values, where a local maximum is always observed, and then converted to a true stress at yielding. If the curves exhibit a less pronounced elasto-plastic transition, e.g. in shear, the Considère construction can be used to determine the yield stress. The yield surfaces are represented with ellipses even though more experimental yield points are required to establish the shape more precisely. In particular, high-triaxiality states of stress like biaxial tension should be investigated to get a better description of the yield surface. The centre of the ellipse does not coincide with the origin as a result of the pressure dependency of the material. Furthermore, their shapes are really similar to the ones found for a rubber-modified PVC [26] and a rubber-modified SAN [27]. The yield loci are expanding with the strain rate which is explained by the strain rate sensitivity of the material, as observed in many other polymers [28,29].

From a modelling point of view, many investigations have been performed to handle pressure dependency of cavitated polymers. Raghava et al. [30] proposed a yield function which was found to well describe the pressure dependency of the yield stress. The model presented by Boyce et al. [31] is also an interesting choice because it accounts for pressure dependency by modifying the

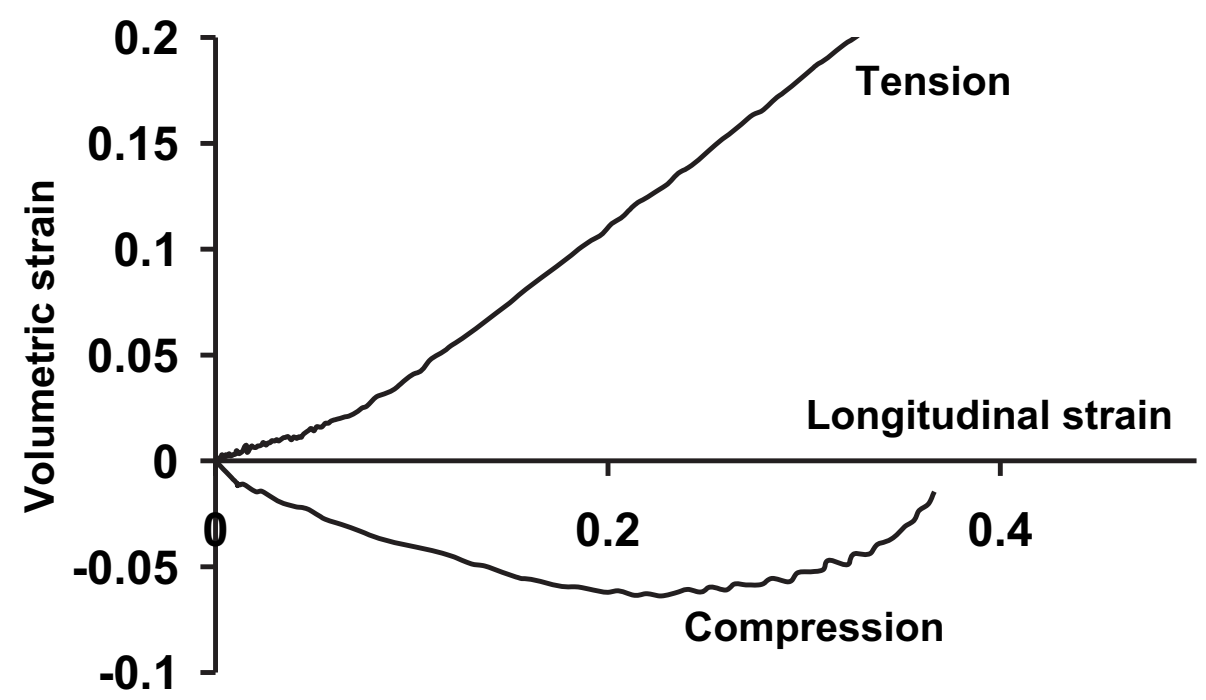

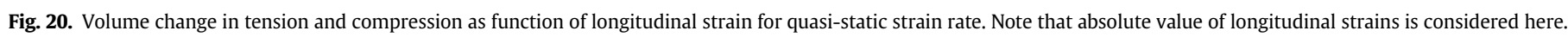


plastic multiplier of the flow rule, and also adding a pressure dependent softening term. On the other hand, the predicted flow was volume preserving, which has been shown not to be correct for the rubber-modified PP investigated in this article.

Still considering the strain-rate sensitivity of the material and its influence on the yield stress, Fig. 19 shows the evolution of the equivalent yield stress as function of equivalent strain rate for the three different states of stress. In this figure, $\sigma_{e q Y}$ is the equivalent yield stress at the considered equivalent strain rate $\dot{\varepsilon}_{e q}$, while $\sigma_{e q 0}$ is the equivalent yield stress at a reference equivalent strain rate $\dot{\varepsilon}_{e q 0}$. The tests performed at the lowest strain rate were taken as the reference cases in all three loading modes. It appears from Fig. 19 that the evolution of the yield stress with strain rate is rather log-linear for the considered range of strain rates between $10^{-3}$ and $102 \mathrm{~s}^{-1}$. Also, it is interesting that application of nominal and true stress measures in tension does not have any significant influence on the results shown in Fig. 19. This means that it does not seem to be necessary to have instrumentation involving digital image correlation for strain rate investigations addressing the yield stress only.

The underlying mechanism of the large rate sensitivity is related to the microstructure of the material. The stretching of the molecule chains during the deformation process is thermodynamically favoured at low strain rates close to the equilibrium conditions. The chains have then the time to stretch. It is particularly true for polymeric materials where molecule chains form a non-ordered net. At higher rates, the chains do not have time to re-organise during deformation, resulting in increased resistance and hence larger observed stresses. It is however unclear why the strain-rate effect seems slightly more pronounced in shear than in tension and compression, which has already been noticed in Section 3.3.

Another strain-rate effect can be observed in the SEM micrograph of fractured specimens in tension shown in Fig. 16b. The fracture surfaces exhibit signs of self-heating, as already observed by van der Waal and Gaymans [23], and this feature does not seem to appear in the quasi-static tests. One assumption could be a thermo-mechanical coupling during deformation and significant adiabatic self-heating of the material [6,7]. Besides, another interesting observation that could confirm such an effect is the shape of the dynamic stress-strain curve in compression that exhibit less strain hardening compared to the quasi-static case. However, this point remains to be confirmed, and more investigations, e.g. using digital infrared thermography, are necessary to establish the underlying mechanisms.

Fig. 20 presents the evolution of the volumetric strain in the quasi-static tension and compression tests. It is clearly seen that the material is dilating in tension and contracting in compression. This observation is in agreement with a cavitated material behaviour observed in tension. It can also be noted that in compression the volume first decreases and then increases again when the longitudinal strain exceeds 0.25 . This increase may be due to the barrelling effect that occurs during testing at large deformations and induces tension area along the surface of the sample and, in particular, where the strains are measured. A SEM study of samples deformed in compression is required to gain more insight into the physical mechanisms occurring at the meso-scale. Similar behaviour has been observed in others cavitated polymers [19,32], and attempts have been made to model this volume changes behaviour using theories developed for porous material. An interesting choice is to use a Gurson-like potential to describe the plastic flow, coupled with a void evolution rule which accounts for cavities growth when the material is stretched. Within this field, Steenbrick et al. [19] and later Pijnenburg et al. [20] modified the Gurson plastic potential to account for large elastic strains occurring in thermoplastics material. Similarly, Zairi et al. [33] also used a modified version of the Gurson potential to account for the damage induced by cavity growth.

\section{Conclusion}

A rubber-particle reinforced polypropylene material was characterised under three states of stress, i.e. tension, compression and shear, and at several strain rates ranging from $10^{-3}$ to $100 \mathrm{~s}^{-1}$. The test results show that the material exhibits strong pressure dependence, and significant volume changes are observed in tension and compression. The mechanical properties are defined by the intrinsic characteristics of the thermoplastic matrix as well as the cavitation processes initiated around particles. It was further found that the flow stress level is highly dependent on the strain rate, and that the rate sensitivity seems to be slightly more pronounced in shear than in tension and compression.

SEM analysis of fracture surfaces in tension shows that the material is highly damaged due to cavity growth. The observed fracture surfaces are typical for polymer blends, and the pattern can be explained by particles debonding and subsequent formation of micro-necks favoured by cavitation during the deformation process. The cavitation process is also confirmed by the evolution of the volume change during tensile and compression tests that were recorded with DIC. It is further recognised that the fracture process seems to be less ductile at high strain rates than under quasi-static conditions. The micrographs reveal that the voids are much smaller in the dynamic case, and there seem also to be some significant selfheating of the material due to the adiabatic regime at high strain rates. This point remains to be confirmed by use of infrared thermography technique.

\section{Acknowledgments}

The authors would like to thank Sonia Achard and Carlos Gomes-Vieira from Renault Materials Engineering Department for their help on the SEM fracture surface analyses.

\section{References}

[1] G'Sell C, Aly-Helal N, Jonas JJ. Effect of stress triaxiality on neck propagation during the tensile stretching of solid polymers. Journal of Materials Science 1983; $18: 1731-42$

[2] Marquez-Lucero A, G'Sell C, Neale KW. Experimental investigation of neck propagation in polymers. Polymer 1989;30:636-42.

[3] Mohanraj J, Barton DC, Ward IM, Dahoun A, Hiver JM, G'Sell C. Plastic deformation and damage of polyoxymethylene in the large strain range at elevated temperatures. Polymer 2006;47:5852-61.

[4] Zhou Y, Mallick PK. Effects of temperature and strain rate on the tensile behaviour of unfilled and talc filled polypropylene. Part I experiments. Polymer Engineering and Science 2002;42:2449-60.

[5] Dasari A, Sarang S, Misra RDK. Strain rate sensitivity of homopolymer polypropylenes and micrometric wollastonite-filled polypropylene composites. Materials Science and Engineering 2004;368:191-204.

[6] Arruda EM, Ahzi S, Li Y, Ganesan A. Rate dependent deformation of semicrystalline polypropylene near room temperature. Journal of Engineering Materials and Technology 1997;119:216-22.

[7] Soong SY, Cohen RE, Boyce MC, Mulliken AD. Rate-dependent deformation behavior of POSS-filled and plasticized poly(vinyl chloride). Macromolecules 2006;39:2900-8.

[8] Bartczak Z, Argon AS, Cohen RE, Weinberg M. Toughness mechanism in semicrystalline polymer blends: I. High density polyethylene toughened with rubbers. Polymer 1998;40:2331-46.

[9] Bartczack Z, Argon AS, Cohen RE, Weinberg M. Toughness mechanism in semicrystalline polymer blends: II. High density polyethylene toughened with calcium carbonate filler. Polymer 1998;40:2347-65.

[10] Bai SL, G'Sell C, Hiver JM, Mathieu C. Polypropylene/polyamide 6/polyethyleneoctene elastomer blends. Part 3. Mechanisms of volume dilatation during plastic deformation under uniaxial tension. Polymer 2005;46:6437-46.

[11] Brough I, Haward RN, Healey G, Wood A. Scanning electron micrographs of high density polyethylene fracture surfaces. Polymer 2004;45:3115-23. 
12 Delhaye V, Clausen AH, Moussy F, Hopperstad OS, Othman R. Mechanical response and microstructural investigation of a mineral and rubber modified polypropylene. Polymer Testing 2010;29:793-802.

[13] Parsons EM, Boyce MC, Parks DM, Weinberg M. Three-dimensional largestrain tensile deformation of neat and calcium carbonate-filled high-density polyethylene. Polymer 2005;46:2257-65.

[14] G'Sell C, Hiver JM, Dahoun A. Experimental characterization of deformation damage in solid polymers under tension, and its interrelation with necking. International Journal of Solids and Structures 2002;39:3857-72.

[15] Zhao H, Abdennadher S, Othman R. An experimental study of square tube crushing under impact loading using a modified large scale SHPB. International Journal of Impact Engineering 2006;32:1174-89.

[16] Bussac MN, Collet P, Gary G, Othman R. An optimisation method for separating and rebuilding one-dimensional dispersive waves from multi-point measurements. Application to elastic or viscoelastic bars. Journal of the Mechanics and Physics of Solids 2002;50:321-49.

[17] G'Sell C, Boni S, Shrivastava S. Application of the plane simple shear test for determination of the plastic behaviour of solid polymers at large strains. Journal of Materials Science 1983;18:903-18.

[18] Othman R, Gueguan P, Challita G, Lebreton D, Pasco F. A modified servohydraulic machine for testing at intermediate strain rates. International Journal of Impact Engineering 2009;36:460-7.

[19] Steenbrink AC, Van Der Giessen E, Wu PD. Void growth in glassy polymers. Journal of the Mechanics and Physics of Solids 1997;45:405-37.

[20] Pijnenburg KGW, Van Der Giessen E. Macroscopic yield in cavitated polymer blends. International Journal of Solids and Structures 2001;38:3575-98.

[21] Galeski A. Strength and toughness of crystalline polymer systems. Progress in Polymer Science 2003;28:1643-99.
[22] Peterlin A. Plastic deformation of unoriented polymer under tensile load. Advances in Polymer Science and Engineering; 1972:1-19.

[23] van der Waal A, Gaymans RJ. Polypropylene-rubber blends. 3. The effect of the test speed on the fracture behaviour. Polymer 1999;40:6045-55.

24] Pawlak A, Galeski A. Plastic deformation of crystalline polymers: the role of cavitation and crystal plasticity. Macromolecules 2005;38:9688-97.

[25] Kody RS, Lesser AJ. Deformation and yield of epoxy networks in constrained states of stress. Journal of Materials Science 1997;32:5637-43.

[26] Crawford E, Lesser AJ. Mechanics of rubber particle cavitation in toughened polyvinylchloride (PVC). Polymer 2000;41:5865-70.

[27] Ramaswamy S, Lesser AJ. Microscopic damage and macroscopic yield in acrylonitrile-butadiene-styrene (ABS) resins tested under multi-axial stress state. Polymer 2003;43:3743-52.

[28] Pessey D, Bahlouli N, Ahzi S, Khaleel A. Strain rate effects on the mechanical response of polypropylene based composites deformed at small strains. Polymer Science - Series A 2008;50:690-7.

[29] Kobayashi A, Ohtani N. Strain rate dependency on stress strain relations of polypropylene. Journal of Applied Polymer Science 2003;15:975-87.

[30] Raghava R, Caddell RM, Yeh GSY. The macroscopic yield behaviour of polymers. Journal of Materials Science 1973;8:225-32.

[31] Boyce MC, Socrate S, Llana PG. Constitutive model for the finite deformation stress-strain behavior of poly(ethylene terephthalate) above the glass transition. Polymer 2000;41:2183-201.

[32] Pawlak A, Galeski A. Cavitation during tensile deformation of polypropylene. Macromolecules 2008;41:2839-51.

[33] Zaïri F, Naït-Abdelaziz M, Woznica K, Gloaguen JM. Constitutive equations for the viscoplastic-damage behaviour of a rubber-modified polymer. European Journal of Mechanics 2005;24:169-82. 